\title{
Efatutazone Dihydrochloride
}

National Cancer Institute

\section{Source}

National Cancer Institute. Efatutazone Dihydrochloride. NCI Thesaurus. Code C64764.

The dihydrochloride salt of efatutazone, an orally bioavailable agonist of peroxisome proliferator-activated receptor gamma (PPAR-gamma) with potential antineoplastic activity. Efatutazone binds to and activates PPAR-gamma, a nuclear hormone receptor and a lig and-activated transcription factor controling gene expression involved in macromolecule metabolism and cell differentiation, specifically adipocyte differentiation. Mediated through activation of PPAR-gamma, this agent is capable of inducing cell differentiation and apoptosis, thereby leading to a reduction in cellular proliferation. 